\title{
ITERATIONS FOR NONLOCAL ELLIPTIC PROBLEMS
}

\author{
EWA SYLWESTRZAK \\ Institute of Mathematics, University of Zielona Góra \\ ul. Szafrana 4a, 65-516 Zielona Góra, Poland \\ E-mail: e.sylwestrzak@im.uz.zgora.pl
}

\begin{abstract}
Convergence of an iteration sequence for some class of nonlocal elliptic problems appearing in mathematical physics is studied.
\end{abstract}

1. Introduction. We consider the following nonlocal elliptic problem:

$$
-\Delta \varphi=M \frac{f(\varphi)}{\left(\int_{\Omega} f(\varphi)\right)^{p}} \quad \text { in } \Omega,
$$

with the homogeneous boundary Dirichlet condition

$$
\left.\varphi\right|_{\partial \Omega}=0 .
$$

Here $\varphi: \Omega \rightarrow \mathbb{R}$ is an unknown function from a bounded domain $\Omega$ of $\mathbb{R}^{n}$ into $\mathbb{R}$, $n \geq 2, f: \mathbb{R} \rightarrow \mathbb{R}^{+}$is a given $C^{1}$ function and $M>0, p>0$ are given parameters.

The physical motivations for the study of nonlocal elliptic problems come from statistical mechanics ([2], [5], [6]), theory of electrolytes $([4])$, and theory of thermistors $([7]$, $[13])$.

If the parameter $p$ equals 1 and the nonlinearity $f(\varphi)$ has the exponential form $e^{-\varphi}$ $\left(e^{\varphi}\right.$, resp.) then (1) is the well-known Poisson-Boltzmann equation and $\varphi$ can be interpreted as the electric (gravitational, resp.) potential of system of particles in thermodynamical equilibrium interacting via Coulomb (gravitational, resp.) potential. In this interpretation, the parameter $M$ is the total charge (mass, resp.) of the particles of the system.

The problem (1)-(2) with given $f(\varphi)$ and $p=2$ appears in modelling the stationary temperature $\varphi$, which results when an electric current flows through a material with temperature-dependent electrical resistivity $f(\varphi)$, subject to a fixed potential difference $\sqrt{M}([7],[13])$.

2000 Mathematics Subject Classification: 35J60, 82C22.

Key words and phrases: nonlinear nonlocal elliptic equation, Picard iteration scheme.

The paper is in final form and no version of it will be published elsewhere. 
The existence and uniqueness of solutions of (1)-(2) depend on the parameters $M, p$, the geometry of the domain $\Omega$, and on some properties of the function $f$. The existence results can be proved using either the technique of sub- and supersolutions ([3]), or variational methods $([6],[8])$, or topological methods $([4],[10],[12],[15])$, whereas the nonexistence results are a consequence of the Pohozaev identity ([3]), or construction of some special subsolutions ([3]).

The existence and uniqueness of solutions for the Poisson-Boltzmann problem with $f(\varphi)=e^{-\varphi}$ and arbitrary $M>0$ have been proved in [8] and [10]. When $f$ has the form $e^{\varphi}$, the solutions do not exist for large $M$, and in general, are not unique. Moreover, the existence and uniqueness depend largely on the geometry of $\Omega$, see [11].

2. Picard iterations for (1)-(2). Our aim is to study the convergence of iteration schemes for nonlocal elliptic problems (1)-(2).

We start with the local elliptic problem

$$
-\Delta \varphi=\lambda f(\varphi) \text { in } \Omega,\left.\quad \varphi\right|_{\partial \Omega}=0,
$$

where $\lambda>0$ is a given constant.

We transform (3) to an integral form

$$
\varphi(x)=\lambda \int_{\Omega} G(x, y) f(\varphi(y)) d y
$$

where $G(x, y)$ is the Green function corresponding to $-\Delta$ and the homogeneous boundary data. The right hand side of (4) defines the operator $T(\varphi)(x)=\lambda \int_{\Omega} G(x, y) f(\varphi(y)) d y$ on the space $C^{0}(\bar{\Omega})$ of continuous functions on $\bar{\Omega}$. It is known that certain assumptions on the function $f$ guarantee that the sequence of iterations $T^{n}(0)$ is convergent in the supremum norm to a minimal solution of (3) ([1], [14]).

The integral form of (1)-(2) is

$$
\varphi(x)=\frac{M}{\left(\int_{\Omega} f(\varphi)\right)^{p}} \int_{\Omega} G(x, y) f(\varphi(y)) d y,
$$

so we introduce the operator $T$ on the space $C^{0}(\bar{\Omega})$ as

$$
T(\varphi)(x)=\frac{M}{\left(\int_{\Omega} f(\varphi)\right)^{p}} \int_{\Omega} G(x, y) f(\varphi(y)) d y .
$$

Any fixed point of $T$ is a solution of (5). We define the Picard iteration scheme for (1)-(2) $\varphi_{n}=T^{n}\left(\varphi_{0}\right)$ by

$$
-\Delta \varphi_{n}=M \frac{f\left(\varphi_{n-1}\right)}{\left(\int_{\Omega} f\left(\varphi_{n-1}\right)\right)^{p}} \quad \text { in } \quad \Omega,\left.\quad \varphi_{n}\right|_{\partial \Omega}=0, \quad n=1,2, \ldots,
$$

and look for a fixed point of $T$ as the limit of the sequence $T^{n}\left(\varphi_{0}\right)$.

First, we note that for a contraction $g$ on the Banach space $X$, i.e. a mapping $g$ : $X \rightarrow X$ such that $\|g(x)-g(y)\| \leq \alpha\|x-y\|$ for some constant $\alpha \in[0,1)$ and for all $x, y \in X, g$ maps the ball $B_{R}(0) \subset X$ into itself whenever $R>\|g(0)\| /(1-\alpha)$.

Indeed, let $R>\|g(0)\| /(1-\alpha)$. Then for $x \in B_{R}(0)$ we have $\|g(x)\| \leq\|g(x)-g(0)\|+$ $\|g(0)\|<\alpha\|x\|+\|g(0)\|<\alpha R+\|g(0)\|<R$.

For the operator $T$ we obtain the following 
Lemma 1. Assume that the function $f$ is Lipschitz continuous and $0<a \leq f \leq b$. Then $T$ is a contraction on $C^{0}(\bar{\Omega})$ for all sufficiently small $M>0$.

Proof. Let $\mu=\left(\int_{\Omega} f(\varphi)\right)^{-p}$. For $\varphi_{1}, \varphi_{2} \in C^{0}(\bar{\Omega})$ we have

$$
\begin{aligned}
\left|T\left(\varphi_{1}\right)(x)-T\left(\varphi_{2}\right)(x)\right| & =M\left|\mu_{1} \int_{\Omega} G(x, y) f\left(\varphi_{1}(y)\right) d y-\mu_{2} \int_{\Omega} G(x, y) f\left(\varphi_{2}(y)\right) d y\right| \\
& \leq M \mu_{1} \int_{\Omega} G(x, y)\left|f\left(\varphi_{1}(y)\right)-f\left(\varphi_{2}(y)\right)\right| d y \\
& +M c(p, \Omega, f) \mu_{1} \mu_{2} \int_{\Omega} G(x, y) f\left(\varphi_{2}(y)\right) d y \int_{\Omega}\left|f\left(\varphi_{1}(y)\right)-f\left(\varphi_{2}(y)\right)\right| d y \\
& \leq M C(p, \Omega, f)\left|\varphi_{1}-\varphi_{2}\right|_{\infty} .
\end{aligned}
$$

We used the fact that $\sup _{x \in \Omega} \int_{\Omega} G(x, y) d y<\infty$ whenever the boundary $\partial \Omega$ is sufficiently smooth, see [9].

For sufficiently small $M$ we have $\alpha_{M}=M C(p, \Omega, f)<1$.

Lemma 2. Assume that $f \in C^{1}(\mathbb{R})$. For a given $R>0$ there exists $\bar{M}$ such that for $M<\bar{M}, T: B_{R}(0) \rightarrow B_{R}(0)$ and $T$ is a contraction.

Proof. Let us define the function $f_{R}$ as $f_{R}(\varphi)=f(\varphi)$ for $|\varphi|<R$ and $f_{R}(\varphi)=f(R)$ for $|\varphi| \geq R$. We denote by $T_{R}$ the operator (6) with $f=f_{R}$.

It follows from Lemma 1 that for sufficiently small $M$ the operator $T_{R}$ is a contraction with the contraction constant less than $1 / 2$. For such $M$ we have $T_{R}: B_{R}(0) \rightarrow B_{R}(0)$. It remains to note that $T_{R}=T$ on $B_{R}(0)$.

From Lemmas 1 and 2 we get

THEOREM 1. If the solutions of (1)-(2) satisfy an a priori estimate $|\varphi|_{\infty}<R$ for $M<$ $M_{0}$, then for sufficiently small $M$ and $\varphi_{0} \in C^{0}(\bar{\Omega})$ with $\left|\varphi_{0}\right|_{\infty}<R$ the Picard iteration sequence $T^{n}\left(\varphi_{0}\right)$ converges to the unique solution of (1)-(2).

As we have seen, the key point of the above reasoning is the existence of an a priori estimate of solutions of (1)-(2). This condition is satisfied for the Poisson-Boltzmann problem of gravitational type in any bounded domain $\Omega$ of $\mathbb{R}^{2}$ and $M<4 \pi$.

In [12] such an a priori estimate of solutions of (1)-(2) has been proved under the assumptions that $f$ is a positive decreasing differentiable function such that $\sup \left|f^{\prime} / f\right|<$ $+\infty$ and $0<p \leq 1$.

3. The iteration scheme for (1)-(2). We have for the local elliptic problem (3) the following fact from the general theory of PDE ([1]).

TheOREM 2. If $f$ is a positive decreasing function, then the problem (3) has a unique solution for each $\lambda>0$.

A modified iteration process for nonlocal elliptic problems has been defined in [15]: $\varphi_{n}=S\left(\varphi_{n-1}\right)=S^{n}\left(\varphi_{0}\right)$, where $S\left(\varphi_{n-1}\right)$ is the unique solution of

$$
\begin{aligned}
& -\Delta \varphi_{n}=\lambda_{n-1} f\left(\varphi_{n}\right) \text { in } \Omega, \\
& \lambda_{n-1}=M\left(\int_{\Omega} f\left(\varphi_{n-1}\right)\right)^{-p},
\end{aligned}
$$




$$
\left.\varphi_{n}\right|_{\partial \Omega}=0, \quad n=1,2, \ldots,
$$

and $\varphi_{0}$ is an arbitrary element of $C^{0}(\bar{\Omega})$.

For this new iteration scheme we have, cf. [15]:

TheOREM 3. If $f$ is a positive decreasing function, $\int_{0}^{\infty} f(s) d s=A<\infty$ and $0<p \leq$ 2 , then for every $\varphi_{0} \in C^{0}(\bar{\Omega})$ the sequence of iterations $S^{n}\left(\varphi_{0}\right)$ is convergent in the supremum norm to a solution of (1)-(2).

Now the question is how this iteration procedure works for (1)-(2) in the case of an increasing function $f$.

4. Iterations for (1)-(2) with $f(\varphi)=e^{\varphi}$. Note that for an arbitrary increasing function $f$ the local elliptic problem (3) may have more than one solution. Therefore, we should modify slightly our process of construction of the iteration sequence for (1)-(2). The idea to use minimal solutions of (3) seems to be reasonable. It is known that the minimal solution of (3) with a positive increasing $f \in C^{1}(\mathbb{R})$ exists and is unique, see [14].

Here we apply the iteration procedure (7)-(9) to the particular case of (1)-(2) with $f(\varphi)=e^{\varphi}, p=1$ and $\Omega=K_{1}(0)=\left\{x \in \mathbb{R}^{2}:|x|<1\right\}$.

In this case (1)-(2) reads

$$
-\Delta \varphi=M \frac{e^{\varphi}}{\int_{\Omega} e^{\varphi}} \quad \text { in } \Omega,\left.\quad \varphi\right|_{\partial \Omega}=0 .
$$

It is known that (10) has a unique radially symmetric solution for $M<8 \pi$, and has no solution for $M \geq 8 \pi([3])$.

The local problem

$$
-\Delta \varphi=\lambda e^{\varphi} \text { in } \Omega,\left.\quad \varphi\right|_{\partial \Omega}=0,
$$

has the minimal solution

$$
\varphi_{1}(r ; \lambda)=\ln \frac{8(4-\lambda-2 \sqrt{4-2 \lambda})}{\left[\lambda+r^{2}(4-\lambda-2 \sqrt{4-2 \lambda})\right]^{2}}
$$

and the maximal solution

$$
\varphi_{2}(r ; \lambda)=\ln \frac{8(4-\lambda+2 \sqrt{4-2 \lambda})}{\left[\lambda+r^{2}(4-\lambda+2 \sqrt{4-2 \lambda})\right]^{2}},
$$

where $\lambda \in(0,2]$, see [1].

We have

$$
\lambda \int_{\Omega} e^{\varphi_{1}}=2 \pi \lambda \int_{0}^{1} r e^{\varphi_{1}(r ; \lambda)} d r=2 \pi(2-\sqrt{4-2 \lambda}) \in(0,4 \pi]
$$

and

$$
\lambda \int_{\Omega} e^{\varphi_{2}}=2 \pi \lambda \int_{0}^{1} r e^{\varphi_{2}(r ; \lambda)} d r=2 \pi(2+\sqrt{4-2 \lambda}) \in[4 \pi, 8 \pi) .
$$

Thus for $M \in(0,4 \pi], \varphi_{1}(r ; \lambda)$ is the solution of (10), where $\lambda$ satisfies

$$
\lambda=M\left(\int_{\Omega} e^{\varphi_{1}}\right)^{-1}=\frac{\lambda M}{2 \pi(2-\sqrt{4-2 \lambda})} .
$$


For $M \in[4 \pi, 8 \pi), \varphi_{2}(r ; \lambda)$ is the solution of (10) with $\lambda$ satisfying

$$
\lambda=M\left(\int_{\Omega} e^{\varphi_{2}}\right)^{-1}=\frac{\lambda M}{2 \pi(2+\sqrt{4-2 \lambda})} .
$$

For $\bar{\varphi} \in C^{0}(\bar{\Omega})$ let $S_{1}(\bar{\varphi})\left(S_{2}(\bar{\varphi})\right.$, resp.) denote the minimal (maximal, resp.) solution of (11) with

$$
\lambda=M\left(\int_{\Omega} e^{\bar{\varphi}}\right)^{-1},
$$

where $M \in(0,4 \pi](M \in[4 \pi, 8 \pi)$, resp.).

For $M \in(0,4 \pi]$ we define the mapping

$$
F_{1}(\lambda)=M\left(\int_{\Omega} e^{\varphi_{1}}\right)^{-1}=\frac{\lambda M}{2 \pi(2-\sqrt{4-2 \lambda})} .
$$

$F_{1}$ is continuous and decreasing for $\lambda \in(0,2]$, has a unique fixed point $\bar{\lambda}=M(8 \pi-$ $M) /\left(8 \pi^{2}\right)$, and $\lim _{\lambda \rightarrow 0^{+}} F_{1}(\lambda)=M / \pi, \lim _{\lambda \rightarrow 2^{-}} F_{1}(\lambda)=M / 2 \pi$. We have $F_{1}^{\prime}(\bar{\lambda})=$ $-M /(8 \pi-2 M)$, hence the fixed point $\bar{\lambda}$ is stable for $M \in(0,8 \pi / 3)$, i.e. $F_{1}^{n}(\lambda) \rightarrow \bar{\lambda}$ for $\lambda$ from some neighbourhood of $\bar{\lambda}$, and unstable for $M \in(8 \pi / 3,4 \pi]$.

The well-defined iterations of a decreasing function may converge to a fixed point or to a periodic orbit of period two.

First, we note that the sequence of iterations $F_{1}^{n}(\lambda), \lambda \in(0,2]$, is defined only for $M \in(0,2 \pi]$, see Fig. 1 .

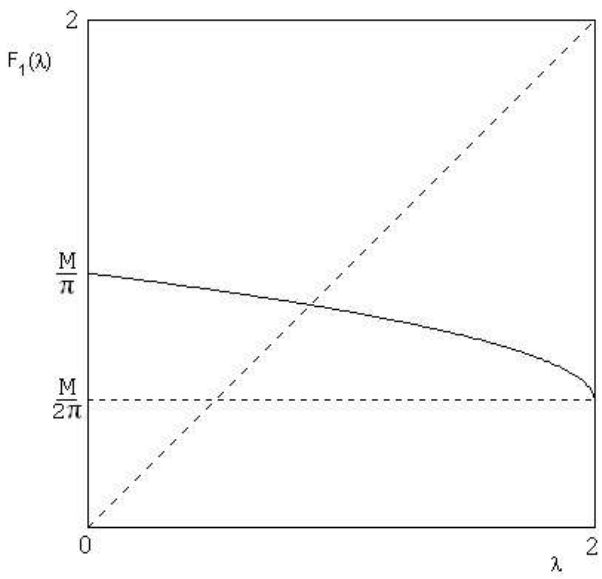

Fig. 1

After some lengthy calculations we observe that $F_{1}^{2}$ has a unique fixed point, and thus there exists no periodic point of $F_{1}$. Hence, taking $M \in(0,2 \pi]$ we get that the iterations $\lambda_{n}=F_{1}^{n}(\lambda)$ tend to a fixed point of $F_{1}$ for any $\lambda \in(0,2]$. This implies that for $\varphi_{0} \in C^{0}(\bar{\Omega})$ satisfying $M\left(\int_{\Omega} e^{\varphi_{0}}\right)^{-1} \leq 2$, the sequence $\varphi_{\lambda_{n}} \equiv \varphi_{1}\left(r ; \lambda_{n}\right)=S_{1}^{n+1}\left(\varphi_{0}\right)$, $n=0,1,2, \ldots, \lambda_{n}=F_{1}^{n}\left(\lambda_{0}\right), \lambda_{0}=M\left(\int_{\Omega} e^{\varphi_{0}}\right)^{-1}$, is convergent in the supremum norm to a solution of $(10)$. 
In fact, using the integral form of (10) we have

$$
\varphi_{\lambda_{n}}(x)=\frac{M}{\int_{\Omega} e^{\varphi_{\lambda_{n-1}}}} \int_{\Omega} G(x, y) e^{\varphi_{\lambda_{n}}(y)} d y, \quad n=1,2, \ldots .
$$

Here, as before, $G(x, y)$ denotes the Green function corresponding to $-\Delta$ and the homogeneous boundary data. Applying the Lebesgue dominated convergence theorem we get

$$
\varphi_{\bar{\lambda}}(x)=\frac{M}{\int_{\Omega} e^{\varphi_{\bar{\lambda}}}} \int_{\Omega} G(x, y) e^{\varphi_{\bar{\lambda}}(y)} d y,
$$

which means that $\varphi_{\bar{\lambda}}$ is a solution of the Poisson-Boltzmann problem (10).

Now for $M \in(2 \pi, 4 \pi]$ we observe that for $M \in(2 \pi, 2 \pi(\sqrt{5}-1)]$ (for $M \in(2 \pi(\sqrt{5}-$ 1), $4 \pi]$, respectively) $F_{1}$ maps the interval $\left[\lambda^{*}, 2\right]\left(\left(\lambda^{*}, \lambda^{* *}\right)\right)$ into itself, see Fig. 2 and Fig. 3. Here $\lambda^{*}=16 \pi(M-2 \pi) / M^{2}$ and $\lambda^{* *}=128 \pi^{2}(M-2 \pi)\left(M^{3}-16 \pi^{2} M+32 \pi^{3}\right) / M^{6}$, they satisfy $F_{1}\left(\lambda^{*}\right)=2$ and $F_{1}\left(\lambda^{* *}\right)=\lambda^{*}$.

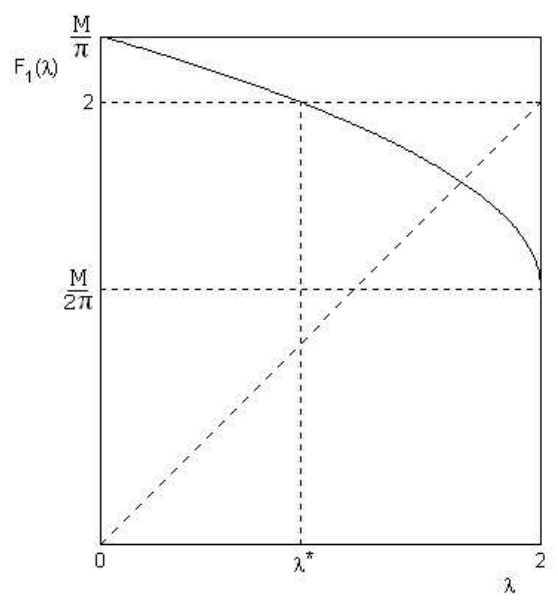

Fig. 2

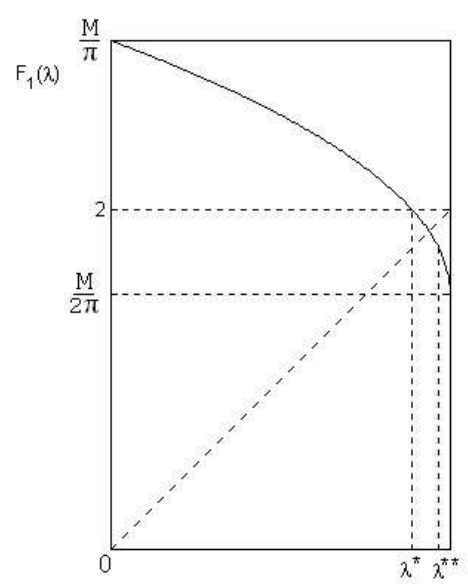

Fig. 3 
When $M \in(2 \pi, 2 \pi(\sqrt{5}-1))$ we get, due to the stability of a fixed point $\bar{\lambda}$ and the absence of periodic points of $F_{1}$, that the sequence of iterations $\lambda_{n}=F_{1}^{n}(\lambda)$ tends to $\bar{\lambda}$ for $\lambda \in\left[\lambda^{*}, 2\right]$. For $M=2 \pi(\sqrt{5}-1)$ the convergence holds on $\left(\lambda^{*}, 2\right)$.

In the case $M \in(2 \pi(\sqrt{5}-1), 8 \pi / 3)$ and $\lambda \in\left(\lambda^{*}, \lambda^{* *}\right)$, some periodic orbit occurs. Moreover, if $M \in(8 \pi / 3,4 \pi)$ then $\bar{\lambda}$ is an unstable fixed point of $F_{1}$, and hence $\varphi_{\lambda_{n}}$ is not convergent to a solution of (10).

Thus, choosing an appropriate initial function $\varphi_{0} \in C^{0}(\bar{\Omega})$, so that $\lambda_{0}=M\left(\int_{\Omega} e^{\varphi_{0}}\right)^{-1}$ lies in the domain of attraction of $\bar{\lambda}$, we can obtain the convergence of the sequence $\varphi_{\lambda_{n}} \equiv S_{1}^{n+1}\left(\varphi_{0}\right)$ to a solution of $(10)$ for $M \in(2 \pi, 2 \pi(\sqrt{5}-1)]$.

Taking $M \in[4 \pi, 8 \pi)$ we get that the map

$$
F_{2}(\lambda)=M\left(\int_{\Omega} e^{\varphi_{2}}\right)^{-1}=\frac{\lambda M}{2 \pi(2+\sqrt{4-2 \lambda})}
$$

is a continuous increasing function of $\lambda \in(0,2]$, has a unique fixed point in $(0,2]$, and $\lim _{\lambda \rightarrow 0^{+}} F_{2}(\lambda)=0, \lim _{\lambda \rightarrow 2^{-}} F_{2}(\lambda)=M / 2 \pi$, see Fig. 4 . Thus the iterations $\lambda_{n}=F_{2}^{n}(\lambda)$ do not tend to a fixed point of $F_{2}$ for any $\lambda \in(0,2]$.

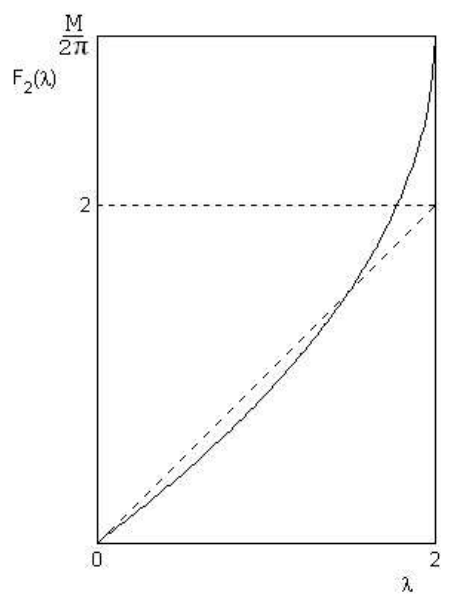

Fig. 4

The conclusion is that for the Poisson-Boltzmann problem of gravitational type we can expect the convergence of our iteration scheme to the solution of this problem only in a restricted range of parameter $M$.

5. Iterations for (1)-(2) with increasing $f$. In this section we study the iteration process for a more general class of problems (1)-(2) with an increasing function $f$.

The construction of the iteration scheme will be based on the theory of minimal solutions of (3). We require the following assumptions on $f$ :

(f1) $f \in C^{1}(\mathbb{R})$;

(f2) $f$ is positive;

(f3) $f$ is increasing. 
The assumptions (f1)-(f3) guarantee the following properties of the minimal solution of (3):

(P1) There exists $\Lambda \in(0,+\infty)$ such that the problem (3) has a unique minimal solution $\widetilde{\varphi}_{\lambda}$ for $\lambda \in(0, \Lambda)([14])$.

(P2) $\widetilde{\varphi}_{\lambda}$ is an increasing function of the parameter $\lambda([14])$.

(P3) $\widetilde{\varphi}_{\lambda}$ is left continuous in $\lambda$, i.e. $\lim _{\lambda \rightarrow \mu^{-}} \widetilde{\varphi}_{\lambda}=\widetilde{\varphi}_{\mu}$ (see Section IV.2.3 in [1]).

(P4) $\lim _{\lambda \rightarrow 0^{+}} \widetilde{\varphi}_{\lambda}=0$.

In order to describe the scheme of iterations for (1)-(2), we define $S(\bar{\varphi})$ for $\bar{\varphi} \in C^{0}(\bar{\Omega})$ as the minimal solution of (3) with

$$
\lambda=M\left(\int_{\Omega} f(\bar{\varphi})\right)^{-p} \equiv H(\bar{\varphi}),
$$

i.e. $S(\bar{\varphi})=\widetilde{\varphi}_{\lambda}$, where $\lambda=H(\bar{\varphi})$. We note that $H$ decreases because of (f3).

We put $\varphi_{0} \equiv 0$. Let $M$ be sufficiently small such that $\lambda_{0}=H(0)<\Lambda$. We take $\varphi_{n}=S^{n}(0)$ as the iteration sequence for (1)-(2) satisfying

$$
-\Delta \varphi_{n}=\lambda_{n-1} f\left(\varphi_{n}\right) \quad \text { in } \Omega, \quad \lambda_{n-1}=H\left(\varphi_{n-1}\right),\left.\quad \varphi_{n}\right|_{\partial \Omega}=0, \quad n=1,2, \ldots .
$$

The question is whether it converges to a solution of $(1)-(2)$.

We have $\varphi_{1}=\widetilde{\varphi}_{\lambda_{0}} \geq 0=\varphi_{0}$, hence $\lambda_{1}=H\left(\varphi_{1}\right) \leq H\left(\varphi_{0}\right)=\lambda_{0}$ which gives $\widetilde{\varphi}_{\lambda_{1}} \leq$ $\widetilde{\varphi}_{\lambda_{0}}$, so $0 \leq \varphi_{2} \leq \varphi_{1}$. The last inequality leads to $\lambda_{2}=H\left(\varphi_{2}\right) \geq H\left(\varphi_{1}\right)=\lambda_{1}$, and thus we get $\varphi_{3}=\widetilde{\varphi}_{\lambda_{2}} \geq \widetilde{\varphi}_{\lambda_{1}}=\varphi_{2}$. On the other hand from $\varphi_{2} \geq 0=\varphi_{0}$ it follows that $\lambda_{2}=H\left(\varphi_{2}\right) \leq H\left(\varphi_{0}\right)=\lambda_{0}$, which implies $\varphi_{3}=\widetilde{\varphi}_{\lambda_{2}} \leq \widetilde{\varphi}_{\lambda_{0}}=\varphi_{1}$. So we have $0 \leq \varphi_{2} \leq \varphi_{3} \leq \varphi_{1}$ and $\lambda_{1} \leq \lambda_{2} \leq \lambda_{0}$. In the same way, from $\varphi_{3} \geq \varphi_{2}$ and $\varphi_{3} \leq \varphi_{1}$ we obtain $\varphi_{4} \leq \varphi_{3}$ and $\varphi_{4} \geq \varphi_{2}$, respectively. Hence $0 \leq \varphi_{2} \leq \varphi_{4} \leq \varphi_{3} \leq \varphi_{1}$ and $\lambda_{1} \leq \lambda_{3} \leq \lambda_{2} \leq \lambda_{0}$ hold.

Continuing, we finally have

$$
\varphi_{0} \leq \varphi_{2} \leq \varphi_{4} \leq \ldots \leq \varphi_{2 k} \leq \varphi_{2 k-1} \leq \ldots \leq \varphi_{3} \leq \varphi_{1} \text { in } \Omega
$$

and

$$
\lambda_{1} \leq \lambda_{3} \leq \lambda_{5} \leq \ldots \leq \lambda_{2 k-1} \leq \lambda_{2 k} \leq \ldots \leq \lambda_{4} \leq \lambda_{2} \leq \lambda_{0}
$$

Thus the sequences $\varphi_{2 k}$ and $\varphi_{2 k-1}$ are convergent uniformly on $\Omega$ to some functions $u$ and $v$, respectively. We see that $u \leq v \leq \varphi_{1}=\widetilde{\varphi}_{\lambda_{0}}$, which gives an a priori estimate of $u$ and $v$, so $|u|_{\infty}<R,|v|_{\infty}<R$, where $R=\left|\widetilde{\varphi}_{\lambda_{0}}\right|_{\infty}$. Hence $u$ and $v$ are small for sufficiently small $M$, because $\lambda_{0}=M\left(\int_{\Omega} f(0)\right)^{-p} \ll 1$ implies via (P4) that $\left|\widetilde{\varphi}_{\lambda_{0}}\right|_{\infty} \ll 1$.

It follows from (13) that $H\left(\varphi_{2 k}\right)=\lambda_{2 k} \rightarrow \check{\lambda}=H(u)$ and $H\left(\varphi_{2 k-1}\right)=\lambda_{2 k-1} \rightarrow \hat{\lambda}=$ $H(v)$. Obviously we have $\hat{\lambda} \leq \check{\lambda} \leq \lambda_{0}$.

Moreover, the functions $u$ and $v$ are solutions of (3) with the parameters $\hat{\lambda}$ and $\check{\lambda}$, respectively, so $u$ and $v$ satisfy

$$
\begin{gathered}
-\Delta u=M \frac{f(u)}{\left(\int_{\Omega} f(v)\right)^{p}} \text { in } \Omega, \\
-\Delta v=M \frac{f(v)}{\left(\int_{\Omega} f(u)\right)^{p}} \text { in } \Omega, \\
\left.u\right|_{\partial \Omega}=0,\left.\quad v\right|_{\partial \Omega}=0 .
\end{gathered}
$$


By (P3) $u$ is a minimal solution, i.e. $u=\widetilde{u}_{\hat{\lambda}}$. Hence to prove the convergence of $\varphi_{n}$ to a solution of (1)-(2), it remains to show that $u=v$, i.e. the problem (14)-(16) does not have a solution $u, v$ in the ball $B_{R}(0) \subset C^{0}(\bar{\Omega})$ such that $u \neq v$. We show that this nonexistence result holds for sufficiently small $M$. First, we note that for $M \ll 1$ the problem (14)-(16) has a unique solution.

Indeed, let us introduce the function space $X=C^{0}(\bar{\Omega}) \times C^{0}(\bar{\Omega})$ with the norm $\|(u, v)\|=|u|_{\infty}+|v|_{\infty}$.

We define the following operator $T$ on $X$

$$
T(u, v)(x)=\left(\frac{M}{\left(\int_{\Omega} f(v)\right)^{p}} \int_{\Omega} G(x, y) f(u(y)) d y, \frac{M}{\left(\int_{\Omega} f(u)\right)^{p}} \int_{\Omega} G(x, y) f(v(y)) d y\right) .
$$

Any fixed point of $T$ is a solution of (14)-(16). Proceeding as in the proof of Theorem 1 , we get that $T$ defined by (17) is a contraction on the ball $B_{R}(0) \subset X$ for sufficiently small $M$.

It remains to note that, whenever the nonlocal elliptic problem (1)-(2) has a solution $\varphi$ satisfying the estimate $|\varphi|_{\infty} \leq C M$, the functions $u=v=\varphi$ solve (14)-(16) and for sufficiently small $M$ it must be a unique solution of this problem.

In this way we have proved the following

TheOrem 4. Let $f$ satisfy (f1)-(f3). Assume that for small $M$ the problem (1)-(2) has a solution $\varphi$ with $|\varphi|_{\infty} \leq C M$. Then for sufficiently small $M$ the iteration sequence $\varphi_{n}=S^{n}(0)$ is convergent in the supremum norm to a solution of (1)-(2).

It is known that if $f$ is a continuous positive increasing function and $\lim _{z \rightarrow+\infty} z / f(z)$ $>M|\Omega|^{-p} \sup _{x \in \Omega} \int_{\Omega} G(x, y) d y$, then (1)-(2) has a solution $\varphi$ which satisfies an a priori estimate $|\varphi|_{\infty} \leq C M$, see [12].

Acknowledgments. The preparation of this paper was partially supported by the EU network HYKE under the contract HPRN-CT-2002-00282. The author would like to thank H. Amann, A. Krzywicki and T. Nadzieja for many helpful suggestions and interesting conversations during the preparation of this paper.

\section{References}

[1] C. Bandle, Isoperimetric Inequalities and Applications, Pitman, Boston, 1980.

[2] F. Bavaud, Equilibrium properties of the Vlasov functional: the generalized PoissonBoltzmann-Emden equation, Rev. Modern Phys. 63 (1991), 129-148.

[3] J. W. Bebernes and A. A. Lacey, Global existence and finite-time blow-up for a class of nonlocal parabolic problems, Adv. Diff. Equations 2 (1997), 927-953.

[4] P. Biler, W. Hebisch and T. Nadzieja, The Debye system: existence and long time behavior of solutions, Nonlinear Analysis T. M. A. 23 (1994), 1180-1209.

[5] P. Biler and T. Nadzieja, Existence and nonexistence of solutions for a model of gravitational interaction of particles, I, Colloq. Math. 66 (1994), 319-334.

[6] E. Caglioti, P. L. Lions, C. Marchioro and M. Pulvirenti, A special class of stationary flows for two-dimensional Euler equations: A statistical mechanics description, Comm. Math. Phys. 143 (1992), 501-525. 
[7] J. A. Carrillo, On a nonlocal elliptic equation with decreasing nonlinearity arising in plasma physics and heat conduction, Nonlinear Analysis T. M. A. 32 (1998), 97-115.

[8] D. Gogny and P. L. Lions, Sur les états d'équilibre pour les densités électroniques dans les plasmas, Math. Modelling and Numerical Analysis 23 (1989), 137-153.

[9] M. Grüter and K. O. Widman, The Green function for uniformly elliptic equations, Manuscripta Math. 37 (1982), 303-342.

[10] A. Krzywicki and T. Nadzieja, Poisson-Boltzmann equation in $\mathbb{R}^{3}$, Ann. Polon. Math. 54 (1991), 125-134.

[11] A. Krzywicki and T. Nadzieja, A note on the Poisson-Boltzmann equation, Zastosowania Matematyki 21 (1993), 591-595.

[12] A. Krzywicki and T. Nadzieja, Nonlocal elliptic problems, Banach Center Publications 52 (2000), 147-152.

[13] A. A. Lacey, Thermal runaway in a nonlocal problem modelling Ohmic heating: Part I: Model derivation and some special cases, Euro. J. Appl. Math. (1995), 129-148.

[14] J. M. Morel and L. Oswald, Remarks on the equation $-\Delta u=\lambda f(u)$ with $f$ nondecreasing, Pitman Res. Notes Math. Ser. 155 (1987), 184-192.

[15] T. Nadzieja and E. Sylwestrzak, Picard iteration scheme for nonlocal elliptic problems with decreasing nonlinearity, Lecture Notes in Nonlinear Analysis 3 (2002), 151-157. 